\title{
ON SOME PROPERTIES OF BINARY RELATIONS
}

\author{
KATUZI ONO
}

Some important notions in the theory of binary relations such as the relative product of two relations and the converse of a relation are defined in Whitehead and Russell's "Principia mathematica" ([1]). McKinsey ([2]) and Tarski ([3]) gave their systems of postulates for the calculus of relations. Ore studied on equivalence relations ([4]) and Riguet on closures (fermeture) of relations ([5] and [6]), and they obtained remarkable results on the structure of these relations. The purpose of this paper is to examine the relative properties of some relations to each other, whose notions are closely related to the notions given by Riguet.

We denote simply by $R S$ the relative product of the relations $R$ and $S$, by $R^{-1}$ the converse of the relation $R$, and by $R[X]$ the set obtained by operating $R$ on the set $X$, i.e. $R$ " $X$ of "Principia mathematica." The notions of the equivalence relation and of the function slightly deviate from the ordinary ones, because a relation is called here an equivalence relation or a function if it is an equivalence relation or a function on their natural domain. In $\S 1$, definitions of these notations and notions and some elementary properties are shortly described as prelimaries.

\section{Elementary properties}

Let $V$ be the universal class. A class $R$ is said to be a relation when and only when $R \subseteq V \times V$. I will write down definitions of fundamental notions and elementary properties without proof in the following:

(1) Def. $R[X]=\{y \mid(E x)(y R x$ and $x \in X)\}$.

(2) $(x)(R[\{x\}]=S[\{x\}]) \Rightarrow R=S$,

i.e. a relation is perfectly determined by its operator character.

( 3 ) $R \cong S \Rightarrow R[X] \subseteq S[X], \quad X \cong Y \Rightarrow R[X] \subseteq R[Y]$.

(4) $R\left[\bigcup_{k \in K} X_{k}\right]=\bigcup_{k \in K} R\left[X_{k}\right], \quad\left(\bigcup_{k \in K} R_{k}\right)[X]=\bigcup_{k \in K} R_{k}[X]$.

Received July 15, 1957. 
(5) $R\left[\bigcap_{k \in K} X_{k}\right] \cong \bigcap_{k \in K} R\left[X_{k}\right], \quad\left(\bigcap_{k \in K} R_{k}\right)[X] \cong \bigcap_{k \in K} R_{k}[X]$.

( 6 ) $(R-S)[X] \supseteqq R[X]-S[X]$.

( 7 ) $\left(\bigcap_{k \in K} R_{k}\right)[\{x\}]=\bigcap_{k \in K} R_{k}[\{x\}], \quad(R-S)[\{x\}]=R[\{x\}]-S[\{x\}]$.

(8) If there is a correspondence $X \rightarrow \varphi(X)$ between subclasses of $V$ such that $\varphi\left(\bigcup_{k \in K} X_{k}\right)=\bigcup_{k \in K} \varphi\left(X_{k}\right)$ always holds, then there exists a relation $R$ for which $R[X]=\varphi(X)$ always holds.

( 9$)(x)(R[\{x\}] \subseteq S[\{x\}]) \Rightarrow R \cong S$.

(10) Def. $R^{-1}=\{\langle x, y\rangle \mid\langle y, x\rangle \in R\}$.

(11) $\left(\bigcup_{k \in K} R_{k}\right)^{-1}=\bigcup_{k \in K} R_{k}^{-1}, \quad\left(\bigcap_{k \in K} R_{k}\right)^{-1}=\bigcap_{k \in K} R_{k}^{-1}, \quad(R-S)^{-1}=R^{-1}-S^{-1}$.

(12) Def. $R S=\{\langle z, x\rangle \mid(E y)[z R y$ and $y S x]\}$.

(13) $(R S)[X]=R[S[X]]$.

(14) $\quad\left(R^{-1}\right)^{-1}=R, \quad(R S) T=R(S T), \quad(R S)^{-1}=S^{-1} R^{-1}$.

(15) Def. $R^{1}=R, \quad R^{2}=R R, \quad R^{3}=R R R, \ldots$

(16) $\quad R^{m} R^{n}=R^{m+n}, \quad\left(R^{n}\right)^{-1}=\left(R^{-1}\right)^{n} . \quad(m, n=1,2, \ldots)$

(17) $R \cong S \Rightarrow R^{-1} \cong S^{-1}, \quad R \cong S \Rightarrow R^{n} \cong S^{n}(n=1,2, \ldots)$,

$R \cong S \Longrightarrow(R T \subseteq S T$ and $T R \cong T S)$.

(18) $R\left(\bigcup_{k \in K} S_{k}\right)=\bigcup_{k \in K} R S_{k}, \quad\left(\bigcup_{k \in K} R_{k}\right) S=\bigcup_{k \in K} R_{k} S$.

(19) $\quad R\left(\bigcap_{k \in K} S_{k}\right) \subseteq \bigcap_{k \in K} R S_{k}, \quad\left(\bigcap_{k \in K} R_{k}\right) S \subseteq \bigcap_{k \in K} R_{k} S$.

(20) $(R-S) T \geqq R T-S T, \quad R(S-T) \supseteqq R S-R T$.

(21) Definition of the identical relation $I: I=\{\langle x, x\rangle \mid x \in V\}$.

(22) $I X=X I=X$.

(23) $(X)[J X=X] \Rightarrow J=I, \quad(X)[X J=X] \Longrightarrow J=I$.

(24) $J \subseteq I \Rightarrow J^{-1}=J^{2}=J$.

(25) $(x)[R \cdot\{\langle x, x\rangle\}=S \cdot\{\langle x, x\rangle\}] \Longrightarrow R=S$, $(x)[\{\langle x, x\rangle\} \cdot R=\{\langle x, x\rangle\} \cdot S] \Rightarrow R=S$.

(26) $R R^{-1} R \supseteqq R, \quad R\left(R^{-1} R \cap I\right)=\left(R R^{-1} \cap I\right) R=R$.

(27) $R(V \times V) R=S(V \times V) S$

$$
\Leftrightarrow\left[R^{-1} R \cap I=S^{-1} S \cap I \text { and } R R^{-1} \cap I=S S^{-1} \cap I\right] \text {. }
$$

(28) Def. $S$ is called symmetric if and only if $S^{-1}=S$.

(29) If $S_{k}$ is symmetric for every $k \in K$, then $\bigcup_{k \in K} S_{k}$ as well as $\bigcap_{k \in K} S_{k}$ are symmetric.

(30) $R \cup R^{-1}, R \cap R^{-1}, R^{-1} R, R R^{-1}$ are symmetric.

(31) Def. $T$ is called transitive if and only if. $T^{2} \cong T$.

(32) If $T$ is transitive, so is also $T^{-1}$. 
(33) If $T$ is transitive and $J \cong I$, then $T \cup J$ is also transitive.

(34) If $T_{k}$ is transitive for every $k \in K$, so is also $\bigcap_{k \in K} T_{k}$.

(35) Def. $F$ is called a function if and only if $F F^{-1} \subseteq I$.

(36) Def. $E$ is called an equivalence relation if and only if $E^{-1} E=E$.

(37) $E$ is an equivalence relation if and only if $E$ is symmetric as well as transitive.

Remark. By the definitions ( 35 ) and (36), " $F$ is a function" means " $F$ is a function on its natural domain," and " $E$ is an equivalence relation" means " $E$ is an equivalence relation of its natural domain, which is equal to its converse domain." For an equivalence relation, reflexivity holds only in the sense $E \supseteqq I \cap\left(E^{-1} E \cup E E^{-1}\right)$.

(38) If $J \subseteq I$, then $J$ is an equivalence relation.

(39) If $E_{k}$ is an equivalence relation for every $k \in K$, then $\bigcap_{k \in K} E_{k}$ is also an equivalence relation.

(40) If $F$ is a function and $F \equiv G$, then $G$ is also a function. In this case holds $F G^{-1}=G F^{-1}=G G^{-1}$.

(41) If $F$ and $G$ are functions, then $F G$ is also a function.

(42) If $F_{k} \cup F_{l}$ is a function for any pair $k, l \in K$, or if $F_{k} F_{l}^{-1} \equiv I$ for any pair $k, l \in K$, then $\bigcup_{k \in K} F_{k}$ is a function.

(43) For any function $F$ hold $(R \cap S) F=R F \cap S F, F^{-1}(R \cap S)=F^{-1} R \cap F^{-1} S$, $(R-S) F=R F-S F$, and $F^{-1}(R-S)=F^{-1} R-F^{-1} S$.

(44) For any equivalence relation $E$ and for any function $F$ hold $E E^{-1} E=E$ and $F F^{-1} F=F$.

(45) If $F$ is a function, then $F^{-1} F$ is an equivalence relation. Conversely, any equivalence relation $E$ can be expressed in the form $E=F^{-1} F$, where $F$ is a function.

(46) Def. $F$ is called an one-to-one correspondence if and only if $F$ and $F^{-1}$ are both functions.

Remark. Evidently hold theorems corresponding to (40)-(44) for one-to-one correspondences.

(47) Def. $P$ is called a partial order if and only if $P$ is transitive and $P \cap P^{-1} \cong I$.

(48) If $P$ is a partial order and $J \subseteq I$, then $(P-I) \cup J$ is also a partial order. 
Remark. By (47), any relation which lies between " $<$ " and " $\leqq$ " is called here a partial order. $P-I$ corresponds to " $<$ ". The linear order $I$, (corresponding to "ฏ”) of the class $C$ can be defined by " $L$ is a linear order of $C$ if and only if $L$ is a partial order and $L \cup L^{-1}=C \times C$."

(49) Def. $R$ is called closed if and only if $R R^{-1} R=R$.

(50) Any function $F$ and any equivalence relation $E$ are closed.

(51) If $R$ is closed, so is also $R^{-1}$. If $R$ and $S$ are closed, so is also $R \cap S$.

(52) For any pair of functions $F$ and $G, F^{-1} G$ is closed. Conversely, any closed relation $R$ can be expressed in the form $R=F^{-1} G$, where $F$ and $G$ are functions.

(53) Def. $\quad \bar{R}=\bigcup_{n=1}^{\infty} R\left(R^{-1} R\right)^{n}$.

(54) $\bar{R} \supseteqq R, \quad \overline{\bar{R}}=\bar{R}$.

(55) $\bar{R}$ is closed, i.e. $\bar{R} \bar{R}^{-1} \bar{R}=\bar{R}$. R is closed if and only if $R=\bar{R}$.

(56) $R \cong S \Rightarrow \bar{R} \subseteq \bar{S}, \quad R \cong S \subseteq \bar{R} \Rightarrow \bar{S}=\bar{R}, \quad \bar{R}^{-1}=\bar{R}^{-1}$.

(57) $\bar{\bigcup}_{k \in K} R_{k} \supseteqq \bigcup_{k \in K} \bar{R}_{k}, \quad \overline{\bigcap_{k \in K}} R_{k} \cong \bigcap_{k \in K} \bar{R}_{k}$.

(58) $\bar{\bigcup}_{k \in K} \bar{R}_{k}=\bigcup_{k \in K} \bar{R}_{k}$.

(59) If $R$ is symmetric, then $\bar{R}=\bigcup_{n=1}^{\infty} R^{n}$ and $\bar{R}$ is an equivalence relation.

(60) $\bar{R}$ is the minimum closed relation including $R$.

(61) $\overline{R^{-1} R}$ and $\overline{R R^{-1}}$ are both equivalence relations.

(62) $\quad \overline{R^{-1} R}=\bar{R}^{-1} \bar{R}=R^{-1} \bar{R}=\bar{R}^{-1} R, \quad \overline{R R^{-1}}=\bar{R} \bar{R}^{-1}=R \bar{R}^{-1}=\bar{R} R^{-1}$.

(63) Def. If $H$ is a relation such that $H[R]$ is a relation for every relation $R$, then $A \cup \bigcup_{n=1}^{\infty} H^{n}[A]$ for any relation $A$ is called the relation recursively generated from $A$ by $H$.

(64) The recursively generated relation from $A$ by $H$ is the minimum relation $F$ satisfying $A \cup H[F] \subseteq F$.

Remark. If we take $A=\{\langle a(\alpha),\langle 0, \alpha\rangle\rangle\}$ and $H=\{\langle\langle b(z, x, \alpha),\langle x+1, \alpha\rangle\rangle,\langle z,\langle x, \alpha\rangle\rangle\rangle \mid x, z \in N\}$, then the condition for $F$ becomes

$$
\begin{aligned}
& f(0, \alpha)=a(\alpha), \\
& f(x+1, \alpha)=b(f(x, a), x, \alpha),
\end{aligned}
$$

where we take $N$ as the set of natural numbers; $F=\{\langle f(x, \alpha),\langle x, \alpha\rangle\rangle \mid x \in N\}$, and $\alpha$ as a finite sequence of parameters. 


\section{Associable system of relations}

The domain and the converse domain of $R$ are classified by the equivalence relations $R^{-1} R$ and $R R^{-1}$ respectively. The relation $R$ is essentially a blockwise one-to-one correspondence between the classified domain of $R$ and the classified converse domain of $R$. I will now define the notion of the associable system of relations, which enables us to study mutual properties between relations of different classified domains and converse domains.

\section{Definition}

(1) A relation $S$ is called an extension of $R$, if and only if $R \cong S$ and $\bar{S} R^{-1} \bar{S} \subseteq \bar{R}$.

(2) A system of relations is called associable, if and only if they have their common extension.

THEOREM 1. A necessary and sufficient condition for two relations $R$ and $S$ to be associable is that the conditions

$$
\begin{aligned}
& (\overline{R \cup S}) R^{-1}(\overline{R \cup S}) \leqq \bar{R}, \\
& (\overline{R \cup S}) S^{-1}(\overline{R \cup S}) \leqq \bar{S}
\end{aligned}
$$

are both satisfied.

Proof. If $T$ is a common extension of $R$ and $S$, then $(R \cup S) R^{-1}(R \cup S)$ $\subseteq \bar{T} R^{-1} \bar{T} \subseteq \bar{R}$. Similarly for the other formula. If the two conditions are satisfied, then $R \cup S$ is a common extension of $R$ and $S$.

THEOREM 2.

(1) Any relation is associable with itself.

(2) Any relation $R$ is associable with $\bar{R}$.

(3) Any relation is associable with its extension.

(4) If $R^{-1} S=R S^{-1}=\varnothing$, i.e. if the domains of $R$ and $S$ as well as their converse domains are disjoint, then $R$ and $S$ are associable.

Proof. (1), (2), (3) Evident.

(4) $(R \cup S) R^{-1}(R \cup S)=\cup V_{0} V_{1}^{-1} V_{2} \ldots V_{2 m-1}^{-1} V_{2 m} R^{-1} W_{0} W_{1}^{-1} W_{2} \ldots W_{2 n-1}^{-1} W_{2}=\bar{R}$, where $V^{\prime}$ 's and $W$ 's stand for $R$ or $S$. Similarly, $(\overline{R \cup S}) S^{-1}(R \cup S)=\bar{S}$. q.e.d.

THEOREM 3. If $R$ and $S$ are associable, so are also $R^{-1}$ and $S^{-1}$.

Froof. Evident. 
THEOREM 4. If a system of $R_{k}$ 's $(k \in K)$ is associable, so is also the system of all $S_{k}$ 's satisfying $R_{k} \leqq S_{k} \leqq \bar{R}_{k}$ for every $k$ in $K$.

Proof. Let $T$ be a common extension of $R_{k}$ 's, i.e. $R_{k} \cong T$ and $\bar{T} R_{k}{ }^{-1} \bar{T} \cong \bar{R}_{k}$. By (55), (56), and (62),

$$
\begin{aligned}
\bar{T} S_{k}{ }^{-1} \overline{T^{N}} \leqq \bar{T} \bar{S}_{k}{ }^{-1} \bar{T} & =\bar{T} \bar{R}_{k}^{-1} \overline{T^{N}}=\bar{T} \bar{R}_{k}^{-1} \bar{R}_{k} \bar{R}_{k}^{-1} \bar{T} \\
& =\bar{T} R_{k}^{-1} \bar{R}_{k} \bar{R}_{k}^{-1} \bar{T} \leqq \bar{T} R_{k}^{-1} \bar{T} \bar{T}^{-1} \bar{T}=\bar{T} R_{k}^{-1} \bar{T} \leqq \bar{R}_{k}=\bar{S}_{k} .
\end{aligned}
$$

Namely, $T$ is also a common extension of all $S_{k}$ 's.

q.e.d.

Theorem 5. If $R$ and $S$ are associable, then

$$
\bar{R} \bar{S}^{-1}=(\bar{R} \cap \bar{S})(\bar{R} \cap \bar{S})^{-1} \text { and } \bar{R}^{-1} \bar{S}=(\bar{R} \cap \bar{S})^{-1}(\bar{R} \cap \bar{S}) .
$$

Proof. Let $\langle x, y\rangle \in \bar{R} \bar{S}^{-1}$, i.e. there be such $z$ that $\langle x, z\rangle \in \bar{R}$ and $\langle y, z\rangle \in \bar{S}$, then

$$
\{\langle x, z\rangle\} \subseteq\{\langle x, z\rangle\}\{\langle y, z\rangle\}^{-1}\{\langle y, z\rangle\} \subseteq(\overline{R \cup S}) \bar{S}^{-1}(\overline{R \cup S}) \subseteq \bar{S},
$$

similarly $\langle y, z\rangle \in \bar{R}$, therefore

$$
\langle x, y\rangle \in(\bar{R} \cap \bar{S})(\bar{R} \cap \bar{S})^{-1} .
$$

We obtain namely $\bar{R} \bar{S}^{-1} \cong(\bar{R} \cap \bar{S})(\bar{R} \cap \bar{S})^{-1}$. On the other hand, evidently $\bar{R} \bar{S}^{-1} \supseteqq(\bar{R} \cap \bar{S})(\bar{R} \cap \bar{S})^{-1}$. Similarly for the other formula. q.e.d.

THeOREM 6. If $P$ and $Q$ resp. $Q$ and $R$ are associable, then

$$
\bar{P}^{-1} \bar{R} \leqq \bar{Q} .
$$

Proof. By the preceding theorem,

$$
\bar{P} \bar{Q}^{-1} \bar{R}=(\bar{P} \cap \bar{Q})(\bar{P} \cap \bar{Q})^{-1} \bar{R} \leqq(\overline{Q \cup R}) \bar{Q}^{-1}(\bar{Q} \cup R) \leqq \bar{Q} . \quad \text { q.e.d. }
$$

THEOREM 7. If all pairs of $R_{k}$ 's are associable, then $\overline{\bigcup_{k \in K} R_{k}}=\bigcup_{k \in K} \bar{R}_{k}$. theorem

Proof. Evidently $\overline{\bigcup_{k \in K} R_{k}} \equiv \bigcup_{k \in K} \bar{R}_{k}$. On the other hand, by the preceding

$$
\begin{aligned}
& \bar{R}_{k_{0}} \bar{R}_{l_{1}}^{-1} \bar{R}_{k_{1}} \bar{R}_{l_{2}}^{-1} \bar{R}_{k_{2}} \ldots \bar{R}_{l_{n-1}}^{-1} \bar{R}_{k_{n-1}} \bar{R}_{l_{n}}^{-1} \bar{R}_{k_{n}} \\
\cong & \bar{R}_{k_{0}} \bar{R}_{l_{1}}^{-1} \bar{R}_{k_{1}} \bar{R}_{l_{2}}^{-1} \bar{R}_{k_{2}} \ldots \bar{R}_{l_{n-1}}^{-1} \bar{R}_{l_{n}} \\
\cdots & \cong \bar{R}_{k_{0}} \bar{R}_{l_{1}}^{-1} \bar{R}_{k_{1}} \bar{R}_{l_{2}}^{-1} \bar{R}_{l_{3}} \\
\leqq & \bar{R}_{k_{0}} \bar{R}_{l_{1}}^{-1} \bar{R}_{l_{2}} \\
\cong & \bar{R}_{l_{1}},
\end{aligned}
$$


so we have $\bigcup_{k \in K} R_{k} \subseteq \bigcup_{k \in K} \bar{R}_{k}$.

q.e.d.

THEOREM 8. A necessary and sufficient condition for a system of relations $R_{k}(k \in K)$ to be associable is that the relations are associable by pairs.

Proof. Necessity is evident. It is also sufficient, because by the preceding theorem

$$
\left(\bigcup_{\jmath \in K} R_{j}\right) R_{k}^{-1}\left(\bigcup_{l \in K} R_{l}\right) \subseteq\left(\bigcup_{j \in K} \bar{R}_{j}\right) \bar{R}_{k}^{-1}\left(\bigcup_{l \in K} \bar{R}_{l}\right)=\bigcup_{\substack{j \in K \\ l \in K}} \bar{R}_{j} \bar{R}_{k}^{-1} \bar{R}_{l} \subseteq \bigcup_{\substack{j \in K \\ l \in K}} \bar{R}_{k}=\bar{R}_{k} \text {. q.e.d. }
$$

THEOREM 9. A necessary and sufficient condition for two equivalence relations $C$ and $D$ to be associable is that $C D=C \cap D$.

Proof. Necessity is evident by (39), (50), and the theorem 5. Conversely: if $C D=C \cap D$, then $(\overline{C \cup D}) C^{-1}(\overline{C \cup D})=\cup P_{1} \ldots P_{m} C^{-1} Q_{1} \ldots Q_{n}=C \cup(C \cap D)$ $=C$, where $P$ 's and $Q$ 's stand for $C$ or $D$. Similarly $(C \cup D) D^{-1}(C \cup D)=D$. Therefore the condition is also sufficient.

\section{Decomposition of the closed relation and of the transitive relation}

Any closed relation can be decomposed into the product of the converse of a function and a function as shown in (52) (Riguet [5], [6]). In this paragraph, this theorem is given in a more refined form in some respect, and some applications of the theorem are given thereafter.

THEOREM 10.

(1) Any closed relation $R$ can be expressed in the form $R=F^{-1} G$, where $F$ and $G$ are functions. (Proposition (52)).

(2) If $R=F^{-1} G$ and $F, G$ are both functions, then generally $R R^{-1} \subseteq F^{-1} F$ and $R^{-1} R \leqq G^{-1} G$. We can find out, however, functions $F_{0}$ and $G_{0}$ out of sub-relations of $F$ and $G$ respectively, for which $R=F_{0}^{-1} G_{0}, R R^{-1}$ $=F_{0}^{-1} F_{0}$, and $R^{-1} R=G_{0}^{-1} G_{0}$.

(3) If $R=F^{-1} G$ and $F, G$ are functions, then $F F^{-1}=G G^{-1}$ if and only if $R R^{-1}=F^{-1} F$ and $R^{-1} R=G^{-1} G$.

Proof. (2) $R R^{-1}=\left(F^{-1} G\right)\left(F^{-1} G\right)^{-1}=F^{-1} G G^{-1} F \leqq F^{-1} I F=F^{-1} F$, similarly $R^{-1} R \subseteq G^{-1} G$. If we put $F_{0}=G G^{-1} F$ and $G_{0}=F F^{-1} G$, then $F_{0} \subseteq I F=F, G_{0} \subseteq I G$ $=G, \quad F_{0}^{-1} G_{0}=F^{-1} G G^{-1} F F^{-1} G=\left(F^{-1} G\right)\left(F^{-1} G\right)^{-1}\left(F^{-1} G\right)=R R^{-1} R=R, \quad F_{0}^{-1} F_{0}$ $=F^{-1} G G^{-1} G G^{-1} F=F^{-1}\left(G G^{-1} G\right)\left(F^{-1} G\right)^{-1}=\left(F^{-1} G\right)\left(F^{-1} G\right)^{-1}=R R^{-1}$, and $G_{0}^{-1} G_{0}$ 
$=G^{-1} F F^{-1} F F^{-1} G=G^{-1}\left(F F^{-1} F\right)\left(F^{-1} G\right)=\left(G^{-1} F\right)\left(F^{-1} G\right)=R^{-1} R$. By $(40), F_{0}$ and $G_{0}$ are both functions. (3) If $R=F^{-1} G, R R^{-1}=F^{-1} F, R^{-1} R=G^{-1} G$, and $F$ and $G$ are functions,

$$
\begin{aligned}
F F^{-1}=I F F^{-1} I \supseteqq G G^{-1} F F^{-1} G G^{-1} & =G\left(F^{-1} G\right)^{-1}\left(F^{-1} G\right) G^{-1} \\
& =G R^{-1} R G=G G^{-1} G G^{-1}=G G^{-1} .
\end{aligned}
$$

Similarly $G G^{-1} \supseteqq F F^{-1}$, so $F F^{-1}=G G^{-1}$. Conversely, if $R=F^{-1} G, F F^{-1}=G G^{-1}$ $\subseteq I$, then

$$
\begin{aligned}
& R R^{-1}=\left(F^{-1} G\right)\left(F^{-1} G\right)^{-1}=F^{-1} G G^{-1} F=F^{-1}\left(F F^{-1} F\right)=F^{-1} F, \\
& R^{-1} R=\left(F^{-1} G\right)^{-1}\left(F^{-1} G\right)=G^{-1} F F^{-1} G=G^{-1}\left(G G^{-1} G\right)=G^{-1} G . \quad \text { q.e.d. }
\end{aligned}
$$

Now, let us take up an equivalence relation $C=F^{-1} F$, where $F$ is a function and $J=F F^{-1}$. The class $K$ of all closed relations $R$, satisfying $R R^{-1}=R^{-1} R=C$, corresponds one-to-one to the class of transformations $T$ on $J$. The one-to-one correspondence is given by $T=F R F^{-1}$ and $R=F^{-1} T F$. If we denote the correspondence by $R \longleftrightarrow T$, we have clearly

(1) $C \longleftrightarrow J$

(2) If $R \longleftrightarrow T$, then $R^{-1} \longleftrightarrow T^{-1}$,

(3) If $R \longleftrightarrow T$ and $S \longleftrightarrow V$, then $R S \longleftrightarrow T V$.

This means that the elements of the class $K$ can be taken as transformations on $C$. In our theory, transformations and one-to-one correspondences are most naturally understood as those on class relations. The theorem 10 shows that any closed relation can be taken as one-to-one correspondence of classes between two systems of classes.

THEOREM 11. If $R$ and $S$ are closed relations and $R \cong S$, then

(1) $R S^{-1} R$ and $S R^{-1} S$ are closed relations satisfying $R \subseteq R S^{-1} R \subseteq S R^{-1} S \subseteq S$,

(2) $R, R S^{-1} R, S R^{-1} S, S$ can be expressed by functions $F, G, F_{0}, G_{0}, F_{1}, G_{1}$, $H$ in such a way that $R=F_{0}^{-1} G_{0}, R S^{-1} R=F_{0}^{-1} H^{-1} H G_{0}, S R^{-1} S=F_{1}^{-1} G_{1}$, and $S=F^{-1} G$, where functions $F, G, F_{0}, G_{0}, F_{1}, G_{1}, H$ satisfy the conditions $F_{0} F_{0}^{-1}=G_{0} G_{0}^{-1}, F_{1} F_{1}^{-1}=G_{1} G_{1}^{-1}=H F_{0} F_{0}^{-1} H^{-1}=H G_{0} G_{0}^{-1} H^{-1}, F F^{-1}$ $=G G^{-1}, F \supseteqq F_{1} \supseteqq H F_{0}, G \supseteqq G_{1} \supseteqq H G_{0}, F F_{1}^{-1} F=F_{1}, G G_{1}^{-1} G=G_{1}$.

Proof. (1) $R=R R^{-1} R \cong R S^{-1} R=R S^{-1} R R^{-1} R \cong S S^{-1} S R^{-1} S=S R^{-1} S \cong S S^{-1} S$ $=S . \quad R S^{-1} R$ is closed, because $\left(R S^{-1} R\right)\left(R S^{-1} R\right)^{-1}\left(R S^{-1} R\right)=R\left(S^{-1} R R^{-1} S R^{-1} R S^{-1}\right) R$ $\subseteq R\left(S^{-1} S S^{-1} S S^{-1} S S^{-1}\right) R=R S^{-1} R ; \quad$ and $S R^{-1} S$ is closed too, because 
$\left(S R^{-1} S\right)\left(S R^{-1} S\right)^{-1}\left(S R^{-1} S\right)=\left(S R^{-1} S S^{-1} R S^{-1} S\right) R^{-1} S \subseteq\left(S S^{-1} S S^{-1} S S^{-1} S\right) R^{-1} S$ $=S R^{-1} S$. (2) By the theorem 10, decompose $S$ into two functions $F, G$ satisfying $S=F^{-1} G, \quad F F^{-1}=G G^{-1}$, which imply $S S^{-1}=F^{-1} F, S^{-1} S=G^{-1} G . \quad G=F S$ and $F=G S^{-1}$, because $F S=F F^{-1} G=G G^{-1} G=G, G S^{-1}=G G^{-1} F=F F^{-1} F=F$. If we put $\hat{G}=F R, \hat{F}=G R^{-1}, G_{1}=\hat{F} S, F_{1}=\hat{G} S^{-1}$, then $F_{1} \supseteqq \hat{F}$ and $G_{1} \supseteqq \hat{G}$, because $F_{1}=\hat{G} S^{-1}$ $=F R S^{-1}=F F^{-1} F R S^{-1}=F S S^{-1} R S^{-1} \supseteqq F S\left(R^{-1} R R^{-1}\right)=F S R^{-1}=G R^{-1}=\hat{F}$, and similarly $G_{1} \supseteqq \hat{G}$. Also $F \supseteqq F_{1}$ and $G \supseteqq G_{1}$, because $F=F F^{-1} F=F S S^{-1} \supseteqq F R S^{-1}$ $=F_{1}$, and similarly $G \supseteqq G_{1}$. Nextly, $\hat{F}^{-1} \hat{G}=R G^{-1} F R=R S^{-1} R$, and $F_{1}^{-1} G_{1}=S \hat{G}^{-1} \hat{F} S$ $=S\left(\hat{F}^{-1} \hat{G}\right)^{-1} S=S R^{-1} S R^{-1} S=S R^{-1} S$; because $S R^{-1} S R^{-1} S=S R^{-1} S$ can be proved as follows: $S R^{-1} S R^{-1} S \supseteqq S R^{-1} R R^{-1} S=S R^{-1} S$, and on the other hand $S R^{-1} S R^{-1} S \cong S S^{-1} S R^{-1} S=S R^{-1} S . \quad \hat{F} \hat{F}^{-1}=\hat{G} \hat{G}^{-1}$, because $\hat{F} \hat{F}^{-1}=G R^{-1} R G^{-1}$ $=G G^{-1} G R^{-1} R G^{-1} G G^{-1}=F\left(F^{-1} G\right) R^{-1} R\left(G^{-1} F\right) F^{-1}=F S R^{-1} R S^{-1} F^{-1} \equiv F R R^{-1} R R^{-1} F^{-1}$ $=F R R^{-1} F^{-1}=\hat{G} \hat{G}^{-1}$, and similarly $\hat{G} \hat{G}^{-1} \equiv \hat{F} \hat{F}^{-1}$. By $(40)$, we obtain $F_{1} F_{1}^{-1}$ $=\hat{G} \hat{G}^{-1}$ and $G_{1} G_{1}^{-1}=\hat{F} \hat{F}^{-1}$ as follows: $F_{1} F_{1}^{-1}=\hat{G} S^{-1} S \hat{G}^{-1}=\hat{G} G^{-1} F F^{-1} G \hat{G}^{-1}$ $=\hat{G} G^{-1} G G^{-1} G \hat{G}^{-1}=\hat{G} G^{-1} G \hat{G}^{-1}=\hat{G} \hat{G}^{-1} \hat{G} \hat{G}^{-1}=\hat{G} \hat{G}^{-1}$, and similarly for the other formula. Accordingly, we have $F_{1} F_{1}^{-1}=G_{1} G_{1}^{-1}=\hat{F} \hat{F}^{-1}=\hat{G} \hat{G}^{-1}$, which implies $\hat{F}^{-1} \hat{F}=\left(R S^{-1} R\right)\left(R S^{-1} R\right)^{-1}$ and $\hat{G}^{-1} \hat{G}=\left(R S^{-1} R^{1}\right)^{-1}\left(R S^{-1} R\right)$. Moreover, we can prove $F_{1}^{-1} F=F_{1}^{-1} F_{1}$ and $G_{1}^{-1} G=G_{1}^{-1} G_{1}$ as follows : $F_{1}^{-1} F_{1} \cong F_{1}^{-1} F=S^{-1} F^{-1} F$ $=S R^{-1} S S^{-1}=S R^{-1}\left(R R^{-1}\right) R\left(R^{-1} S S^{-1}\right) \subseteq S R^{-1} S S^{-1} R S^{-1}=\mathrm{SR}^{-1} F^{-1} F R S^{-1}=F_{1}^{-1} F_{1}$, and similarly for the other formula. Accordingly, by making use of (40), we get $F F_{1}^{-1} F=F_{1} F_{1}^{-1} F=F_{1} F_{1}^{-1} F_{1}=F_{1}$ and similarly $G G_{1}^{-1} G=G_{1}$.

Now, by the theorem $10, R$ can be decomposed into two functions $F_{0}, G_{0}$ satisfying $R=F_{0}^{-1} G_{0}, F_{0}^{-1} F_{0}=G_{0}^{-1} G_{0}$, which imply $R R^{-1}=F_{0}^{-1} F_{0}, R^{-1} R=G_{0}^{-1} G_{0}$. Let us define the relation $H$ by $H=\hat{F} F_{0}^{-1} . \quad H$ is a function, because $H H^{-1}=\hat{F} F_{0}^{-1} F_{0} \hat{F}^{-1}=\hat{F} R R^{-1} \hat{F}^{-1} \cong \hat{F}\left(R S^{-1} R\right)\left(R S^{-1} R\right)^{-1} \hat{F}^{-1}=\hat{F} \hat{F}^{-1} \hat{G} \hat{G}^{-1} \hat{F} \hat{F}^{-1}$ $=\hat{F} \hat{F}^{-1} \hat{F} \hat{F}^{-1} \hat{F} \hat{F}^{-1}=\hat{F} \hat{F}^{-1} \cong I$; and $H=\hat{G} G_{0}^{-1}$, because $\hat{G} G_{0}^{-1}=\hat{G} \hat{G}^{-1} \hat{G} G_{0}^{-1} G_{0} G_{0}^{-1}$ $=\hat{F} \hat{F}^{-1} \hat{G} G_{0}^{-1} F_{0} F_{0}^{-1}=\hat{F} R S^{-1} R R^{-1} F_{0}^{-1}=\hat{F}\left(R S^{-1} R\right)\left(R S^{-1} R\right)^{-1} F_{0}^{-1}=\hat{F} \hat{F}^{-1} \hat{F} F_{0}^{-1}=\hat{F} F_{0}^{-1}$ $=H$, as $R S^{-1} R R^{-1}=\left(R S^{-1} R\right)\left(R S^{-1} R\right)^{-1}$ is easily provable. Lastly, we can obtain $\hat{F}=H F_{0}, \hat{G}=H G_{0}$ as follows : $H F_{0}=\hat{F} F_{0}^{-1} F_{0}=\hat{F} R R^{-1}=\left(G R^{-1}\right) R R^{-1}=G R^{-1}=\hat{F}$, and similarly for the other formula.

q.e.d.

THEOREM 12. If a transitive relation $T$ satisties the condition $T \cap T^{-1}$ $\supseteq\left(T T^{-1} \cup T^{-1} T\right) \cap I^{1)}$ then $T$ can be expressed in the form $T=F^{-1} S F$, where

1) By (33), we can find out for any transitive relation $S$, a transitive relation $T=S U \mathrm{~J}$, which satisfies the condition. 
$F$ is a function and $S$ is a partial order, i.e. $S^{2} \subseteq S$ and $S \cap S^{-1} \cong I$.

Proof. If we take $H=T \cap T^{-1}, H$ is an equivalence relation including $\left(T T^{-1} \cup T^{-1} T\right) \cap I$, because evidently $H^{-1}=H$ and $H^{2}=\left(T \cap T^{-1}\right)^{2}$ $\cong T^{2} \cap\left(T^{-1}\right)^{2} \cong T \cap T^{-1}$. By (45), there is a function $F$ such that $H=F^{-1} F$. If we put $S=F T F^{-1}$, then $T=F^{-1} S F$; because $T \supseteqq T^{2} \supseteqq T H \supseteqq T\left(T^{-1} T \cap I\right)$ $=T$ by (26), and similarly $T=H T$, so $T=H T H=F^{-1} F T F^{-1} F=F^{-1} S F$.

Now I prove that $S$ is a partial order. Firstly, $S^{2}=F T F^{-1} F T F^{-1}$ $=F T H T F^{-1} \cong F T T T F^{-1} \cong F T F^{-1}=S$. Secondly, $F F^{-1} S=F F^{-1} F T F^{-1}=F T F^{-1}$ $=S$ and similarly $S F F^{-1}=S, F F^{-1} S^{-1}=S^{-1} F F^{-1}=S^{-1}$. As $F F^{-1}$ and $F$ are functions, we can compute by (43) as follows: $S \cap S^{-1}=F F^{-1} S \cap F F^{-1} S^{-1}$ $=F F^{-1}\left(S \cap S^{-1}\right)=F F^{-1}\left(S F F^{-1} \cap S^{-1} F F^{-1}\right)=F F^{-1}\left(S \cap S^{-1}\right) F F^{-1}=F\left(F^{-1} S \cap F^{-1} S\right) F F^{-1}$ $=F\left(F^{-1} S F \cap F^{-1} S^{-1} F\right) F^{-1}=F\left(T \cap T^{-1}\right) F^{-1}=F H F^{-1}=F F^{-1} F F^{-1}=F F^{-1} \subseteq I$.

$$
\text { q.e.d. }
$$

By this theorem, we can see that any transitive relation $T$ is essentially a partial order on the classes classified by a suitable equivalence relation.

\section{REFERENCES}

[1] A. N. Whitehead and B. Russell, Principia mathematica, vol. 1, Cambridge University Press, 1925 (2nd ed.).

[2] J. C. C. McKinsey, Postulates for the calculus of binary relations, Journ. of Sym. Log., vol. 5 (1940), pp. 85-97.

[3] A. Tarski, On the calculus of relations, Journ. of Sym. Log., vol. 6 (1941), pp. 73-89.

[4] O. Ore, Theory of equivalence relations, Duke Math. Journ., vol. 9 (1942), pp. 573-627.

[5] J. Riguet, Relations binaires, fermetures, correspondances de Galois, Buletin de Société Mathematique de France, vol. 79 (1948), pp. 114-155.

[6] J. Riguet, Quelques propriétés des relations difonctionnelles, Comptes rendus hebmodaires des séances de l'Academie des Sciences, vol. 230 (1950), pp. 1999-2000.

\section{Mathematical Institute}

Nagoya University 